Diabetesinzidenz: Einen Monat nach Absetzen von Insulinglargin wurden bei 25\% (Glargingruppe) bzw. bei 32\% (Kontrollgruppe) ein neu aufgetretener Typ2-Diabetes diagnostiziert (relative Risikoreduktion: $28 \%)$.

Dem gegenüber stand eine relativ geringe Zunahme von Hypoglykämien im Glarginarm ( 1 vs. $0,3 \%$ pro 100 Patientenjahre). Patienten mit Insulintherapie nahmen in sechs Jahren im Schnitt 1,6 kg an Gewicht $\mathrm{zu}$, Kontrollpatienten $0,5 \mathrm{~kg}$ ab.

Insulinglargin sei mit diesen Daten das am besten untersuchte Arzneimittel in der antidiabetischen Therapie, betonte Studienleiter Hertzel Gerstein, Professor für Endokrinologie an der MacMaster University in Hamilton/Kanada. Zwar habe sich die präventive Wirkung auf kardiovaskuläre Ereignisse nicht belegen lassen. Dennoch wertet Gerstein die Studie als Erfolg: Nach 90 Jahren Insulintherapie, die lange Zeit von Unsicherheiten über mögliche ungünstige Effekte begleitet waren, habe man nun für Glargin die beruhigende Gewissheit, dass von einem Insulin auch langfristige keine negativen Auswirkungen auf wichtige Aspekte der Gesundheit zu befürchten seien.

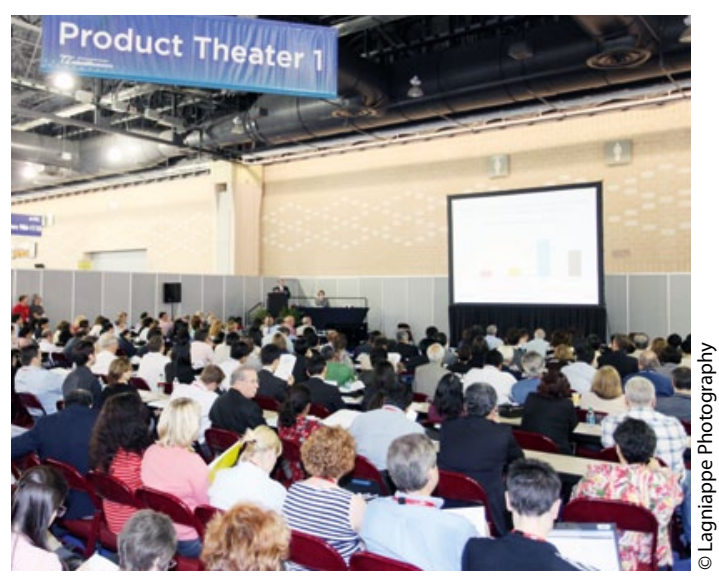

Sowohl spannende Neuigkeiten als auch solides Basiswissen wurden beim ADA 2012 vermitteln.

\section{„Fischöl“ kann Herztod nicht verhindern}

Ebenfalls in der ORIGIN-Studie wurde untersucht, ob die tägliche Nahrungsergänzung mit 1 g Omega3-Fettsäuren im Placebovergleich (Olivenöl) die Rate kardiovaskulärer Ereignisse senken kann. Doch die Maßnahme erwies sich als ungeeignet: Fischöl senkte zwar die Triglyzeride um 14,5 mg/dl, hatte aber keinen Einfluss auf klinische Endpunkte (s.a. rechts).

Peter Overbeck

Quelle: MMW - Fortschritte der Medizin 2012; 154 (12): 24 basierend auf: Basal Insulin and Cardiovascular and Other Outcomes in Dysglycemia, NEJM online 11. Juni 2012, präsentiert beim Kongress der ADA in Philadelphia, 11. Juni 2012

\section{Kurz notiert}

\section{Prävention mit „Fischöl“ kann Herztod nicht verhindern}

Eine tägliche Nahrungsergänzung mit $1 \mathrm{~g}$ Nahrungsergänzung mit Omega-3-Fettsäuren („Fischöl"-Kapseln) senkte in der ORIGIN-Studie zwar die Triglyzeridwerte der Patienten - von der erhofften Reduktion der kardiovaskulären Mortalität bei Typ-2-Diabetikern oder Menschen mit hohem Diabetesrisiko war aber auch nach bis zu sieben Jahren Nachbeobachtung nichts zu sehen.

Studienteilnehmer hatten Diabetes oder Prädiabetes In ORIGIN (Outcome Reduction with an Initial Glargine Intervention) ist parallel der mögliche präventive Effekt von zwei Therapiestrategien auf kardiovaskuläre Ereignisse geprüft worden: Zum einen die des Basalinsulins Insulinglargin (Beitrag links), zum anderen die einer Nahrungsergänzung mit Omega-3-Fettsäuren (vulgo: „Fischöl“).

Zur Prüfung der Supplementierungsstrategie sind 12536 Personen mit kardiovaskulären Risikofaktoren und Typ2-Diabetes (82\% der Studienpopulation) oder leichteren Störungen des Glukosemetabolismus wie gestörter Nüchternblutzucker (IFG) oder gestörte Glukosetoleranz (IGT) randomisiert einer Therapie mit Omega-3-Fettsäuren (ein Gramm pro Tag, bestehend aus 465 mg Eicosapentaensäure und 375 mg Docosahexaensäure) oder mit Placebo (Olivenöl) zugeteilt worden. Der primäre Endpunkt der Studie war die Rate der kardiovaskulär bedingten Todesfälle.

\section{Kein Effekt auf kardiovaskuläre Mortalität}

Die gleichzeitig im „New England Journal of Medicine“ publizierten Ergebnisse hat Dr. Jackie Bosch aus Hamilton/ Kanada auch beim Kongress der American Diabetes Association (ADA) in Philadelphia vorgestellt.

Die Bilanz der Supplementierung nach rund sechs Jahren Nachbeobachtung: Mit 9,1\% (Omega-3-Fettsäuren) und 9,3\% (Placebo) waren die Raten für die kardiovaskuläre Mortalität nahezu gleich und nicht signifikant unterschiedlich.

Triglyzeride um 14,5 mg/dl gesenkt Auch an den Gesamtraten aller schwerwiegenden kardiovaskulären Ereignisse (16,5 versus 16,3\%) war kein Effekt der Supplementierung abzulesen. Gleiches gilt für die Endpunkte Gesamtsterberate (15,1 versus 15,4\%) und Tod infolge Arrhythmien (4,6 versus 4,1\%).

Einen Effekt hatte die Supplementierung von Omega-3Fettsäuren allerdings doch: Die Triglyzeridwerte wurden damit im Schnitt um 14,5 mg/dl stärker gesenkt als durch Placebo - was jedoch augenscheinlich ohne jede protektive Wirkung auf Herz und Gefäße geblieben war.

Dr. med. Jochen Aumiller 